\title{
Programmable integrated front-end for SiPM/PMT PET detectors with continuous scintillating crystal
}

\author{
V. Herrero-Bosch, ${ }^{1}$ J.M. Monzó, A. Ros, R.J. Aliaga, A. González, C. Montoliu, \\ R.J. Colom-Palero and J.M. Benlloch \\ Instituto de Instrumentación para Imagen Molecular (I3M), \\ SIC - Universitat Politècnica de València - CIEMAT, \\ Camino de Vera s/n, 46022 Valencia, Spain \\ E-mail: viherbos@i3m.upv.es
}

ABSTRACT: AMIC architecture has been introduced in previous works in order to provide a generic and expandable solution for implementing large number of outputs SiPM array/PMT detectors. The underlying idea in AMIC architecture is to calculate the moments of the detected light distribution in an analog fashion. These moments provide information about energy, $x / y$ position, etc. of the light distribution of the detected event. Moreover this means that a small set of signals contains most of the information of the event, thus reducing the number of channels to be acquired.

This paper introduces a new front-end device AMIC2GR which implements the AMIC architecture improving the features of the former integrated devices. Higher bandwidth and filtering coefficient precision along with a lower noise allow to apply some detector enhancements. Inhomogeneity among detector elements throughout the array can be reduced. Depth of interaction measurements can be obtained from the light distribution analysis. Also a common trigger signal can be obtained for the whole detector array. Finally AMIC2GR preamplifier stage close to SiPM output signals optimizes signal to noise ratio, which allows to reduce SiPM gain by using lower operating voltages thus reducing dark noise.

KEYWORDS: Analogue electronic circuits; Front-end electronics for detector readout; Gamma camera, SPECT, PET PET/CT, coronary CT angiography (CTA)

\footnotetext{
${ }^{1}$ Corresponding author.
} 


\section{Contents}

1 Introduction 1

2 AMIC architecture $\quad 1$

3 AMIC2GR improvements 2

4 Detector enhancement applications 3

4.1 Gain equalization 3

4.2 SiPM array operating voltage optimization 5

4.3 SiPM array trigger signal generation $\quad 5$

5 Conclusions 6

\section{Introduction}

Detectors based on continuous scintillating crystals and SiPM arrays have demonstrated to be a high performance alternative to position sensitive PMT [1]. However SiPM arrays introduce new problems compared to former PMT technology such as a higher dark count or the lack of a common trigger signal. In order to overcome most of these problems and provide a generic and expandable solution for implementing any size output array based detectors, an integrated front-end AMIC was presented in [2].

A new integrated front-end device AMIC2GR has been developed which improves several features of the original AMIC. In section 2 a review of AMIC architecture is reported. Section 3 explains AMIC2GR improvements and shows test results related to its electrical performance. Section 4 shows functional results of possible detector enhancements obtained by using a AMIC2GR based front-end. Finally some conclusions are stated in section 5 .

\section{AMIC architecture}

The underlying idea in AMIC architecture is to calculate the moments of the detected light distribution in an analog fashion. Due to the additive nature of the moment calculation, the operation can be carried out on a single device or split it into several devices, adding the partial results afterwards. Up to eight different moments can be calculated, thus obtaining information about energy, $\mathrm{x} / \mathrm{y}$ position, depth of interaction $[2,3]$ or any other parameter related to the light distribution of the detected event.

The Input Stage (IS) is composed by a set of 64 input preamplifiers which isolate the sensor outputs from the Computational Blocks (CB) where the moments are calculated. As shown in figure $1 \mathrm{~b}$ each preamplifier generates eight identical copies of the input currents which are fed into 

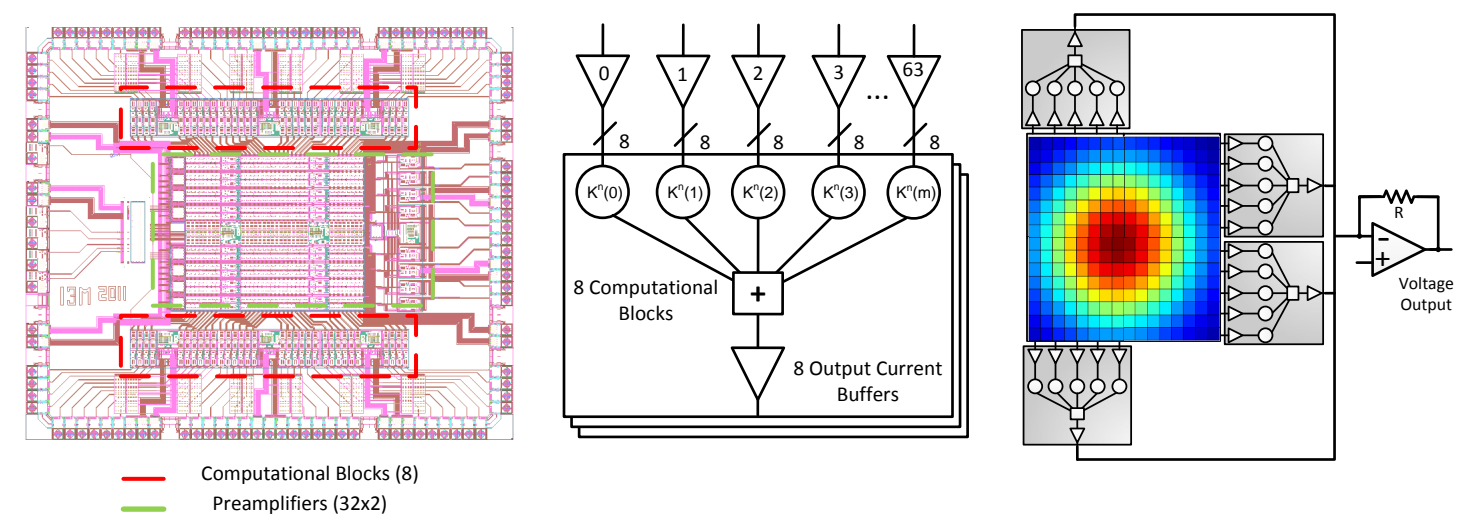

Figure 1. AMIC2GR layout (Technology AMSC35 - $4.22 \mathrm{~mm} \times 3.58 \mathrm{~mm}$ ) (a). Functional Schematic of AMIC2GR (b). Expansion example of AMIC architecture (c).

the $\mathrm{CB}$. The most important specifications of these preamplifers are input capacity range and noise. The wide range of parasitic capacitance of SiPM and PMT can reduce bandwidth or introduce stability issues in feedback structures. Noise may limit resolution in sensors with a high number of outputs.

The CB implements a weighted sum of the input currents from the sensor, which is a spatially discretized version of equation 2.1, where $l(x, y)$ is the light distribution on detecting surface. Depending on the $K^{n}(x, y)$ coefficients different properties of the light distribution can be extracted [4].

$$
C B n_{\text {out }}=\iint K^{n}(x, y) l(x, y) \mathrm{d} x \mathrm{~d} y
$$

All the operations are carried out in current mode, and the weighting operations are implemented using linear MOS current dividers [5]. Weighting coefficients are programmed through a serial interface and stored in local static memory cells. Finally all the weighted currents are added together and introduced in an output current buffer with a programmable offset compensation which helps to reduce bias currents from all previous stages. An example of expansion of the number of inputs of the front-end is included in figure 1c. Current mode operation along the whole architecture also helps to reduce additional external electronics.

\section{AMIC2GR improvements}

The preamplifier stage has been completely redesigned in order to guarantee SiPM compatibility, providing an expanded bandwidth and an increased speed when using these devices. Results are now comparable to those obtained with PMT. The preamplifier design (figure 2) makes use of the pole splitting effect introduced by Miller compensation $\left(C_{M}\right)$ to push the secondary pole introduced by input parasitic capacitance to higher frequencies thus increasing phase margin. A high signal gain between points $\mathrm{A}$ and $\mathrm{B}$, shown in figure 2, maximizes the shift of the secondary pole.

Electrical simulation results related to the preamplifier are shown in table 1 for two typical photomultipliers (SiPM and PMT). Gain linear error is due to mismatch among different pream- 


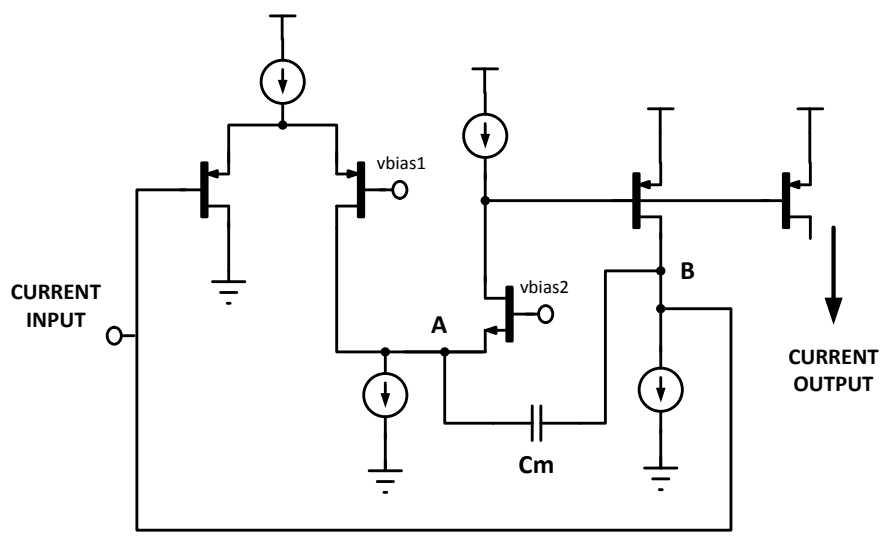

Figure 2. Preamplifier design.

Table 1. Preamplifier characteristics.

\begin{tabular}{|r|c|c|}
\hline & MPPC S10362-11 & PMT H8500 \\
\hline Bandwidth & $40 \mathrm{MHz}$ & $70 \mathrm{MHz}$ \\
Noise & $27.9 \mathrm{pA} / \sqrt{\mathrm{Hz}}$ & $18.3 \mathrm{pA} / \sqrt{\mathrm{Hz}}$ \\
Gain Linear Error & $<3 \%$ & $<3 \%$ \\
\hline
\end{tabular}

plifiers and can be compensated through calibration. A low noise performance has been achieved which yields Signal to Noise ratios (SNR) of $60 \mathrm{~dB}^{1}$.

Precision of the coefficients applied to the detector output currents have been improved. The mean Effective Number of Bits of the coefficients (ENOB) is now 7.9 bits with a worst case of 7.3 bits, which means that the nominal precision ( 8 bits) is achieved in almost every coefficient.

\section{Detector enhancement applications}

An AMIC2GR based front-end can improve detector performance in different ways and allows to deal with specific problems introduced by SiPM arrays. In this section some possible applications are shown. Measurements have been carried out with PMT (H8500) and 64 SiPM array (S1036211) coupled to a LSO continuous scintillating crystal in a dual detector setup (figure 3) operating in coincidence mode.

\subsection{Gain equalization}

Coefficient values of the computational blocks may include a gain correction in order to equalize detector response. A simple method has been proposed which gives the correction factors based on a set of 64 equally distributed measurements on detector surface and an ideal Light Distribution Model (LDM) shown in figure $3 \mathrm{a}$ and figure $3 \mathrm{~b}$. The set of equations in 4.1 show the relationship between 64 photopeak measurements $P P_{i, j}$, weights associated to LDM $W_{n, m, i, j}$ and a light gain

\footnotetext{
${ }^{1}$ Measured with PMT Hamamatsu H8500
} 


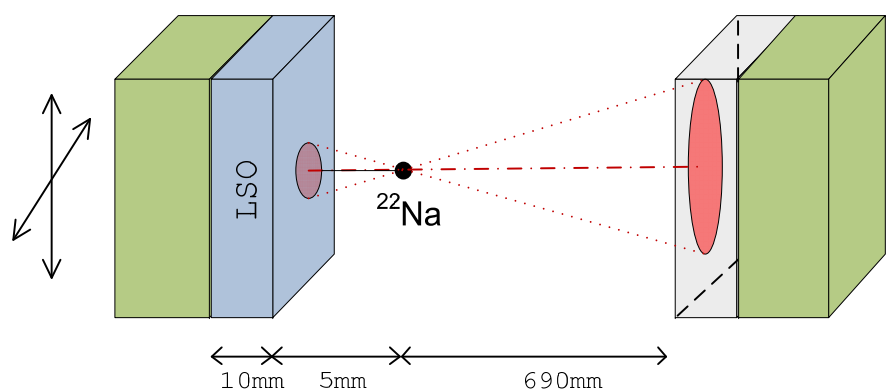

(a)

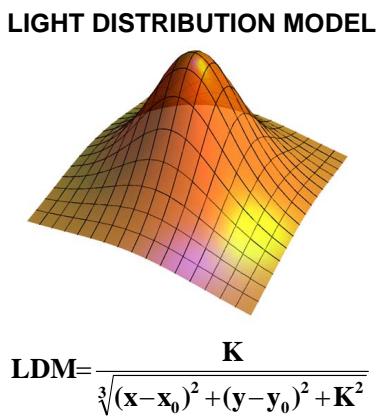

(b)

Figure 3. Setup Configuration (a) and Light Distribution Model (b).
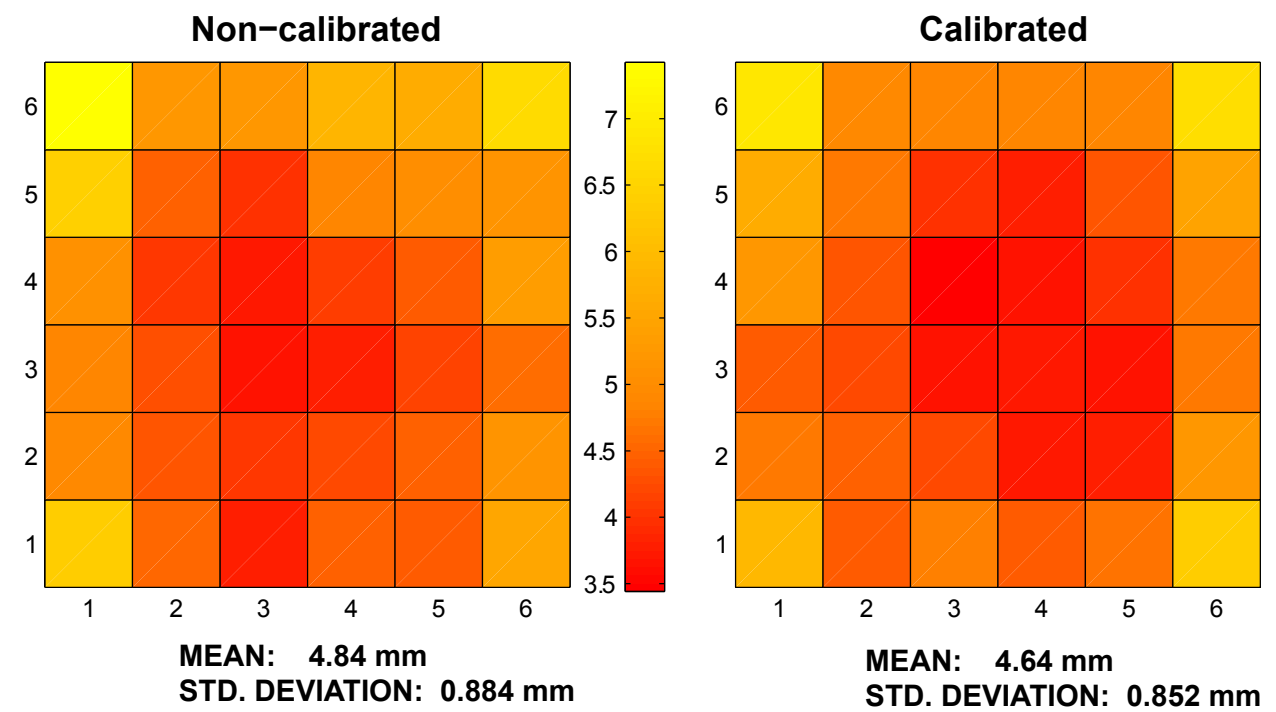

Figure 4. Combined X-Y resolution (H8500 Hamamatsu).

factor $L G_{i, j}$ to be determined which includes the gain spread. After solving the equation system, $L G_{i, j}$ matrix is obtained which just after normalization provides the desired correction factors.

Setup structure shown in figure 3 a has been also used to take 36 equally distributed measurements on the equalized detector surface (the closest to the point source) which are non-coincident with the ones used in the calibration procedure. Both energy and spatial resolution show an improvement in mean values and a lower deviation over the total area (figure 4 and figure 5). Also a more homogeneous behavior is observed specially in energy resolution.

$$
\begin{aligned}
W_{0,0,0,0} \cdot L G_{0,0}+W_{0,1,0,0} \cdot L G_{0,1}+\ldots+W_{7,7,0,0} \cdot L G_{7,7} & =P P_{0,0} \\
W_{0,0,0,1} \cdot L G_{0,0}+W_{0,1,0,1} \cdot L G_{0,1}+\ldots+W_{7,7,0,1} \cdot L G_{7,7} & =P P_{0,1} \\
\ldots & \\
W_{0,0,7,7} \cdot L G_{0,0}+W_{0,1,7,7} \cdot L G_{0,1}+\ldots+W_{7,7,7,7} \cdot L G_{7,7} & =P P_{7,7}
\end{aligned}
$$



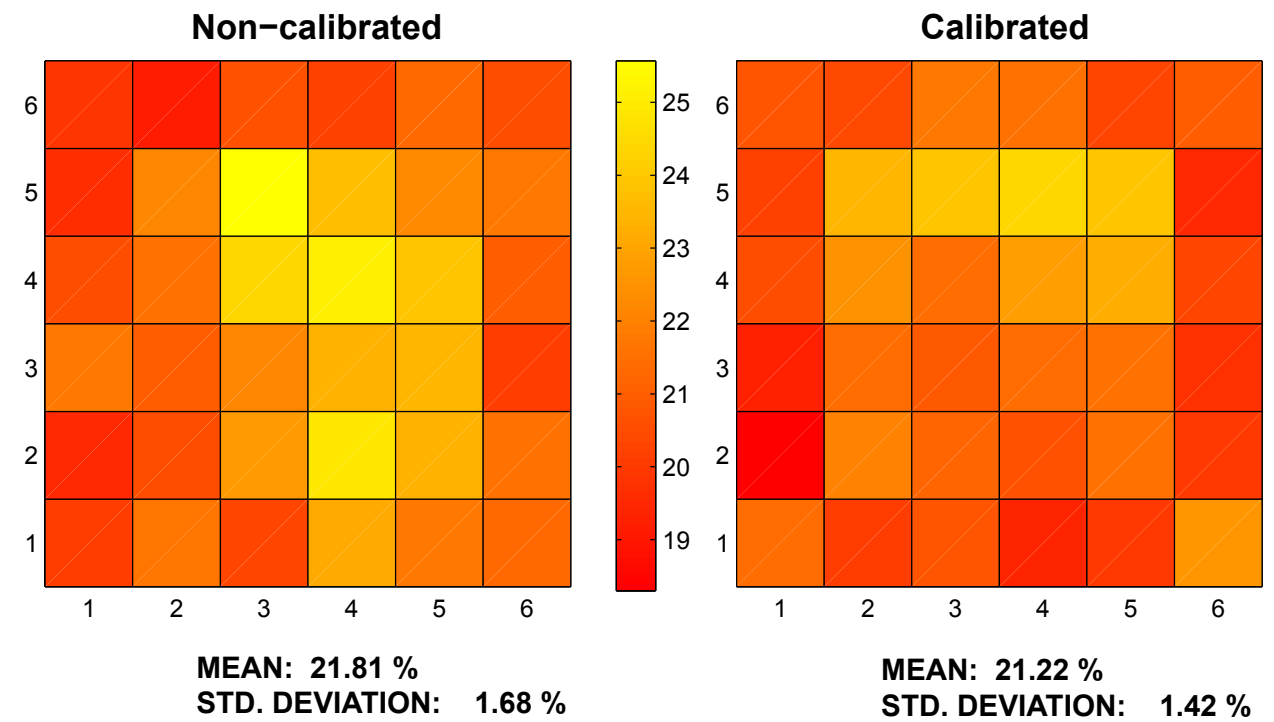

Figure 5. Energy resolution (H8500 Hamamatsu).
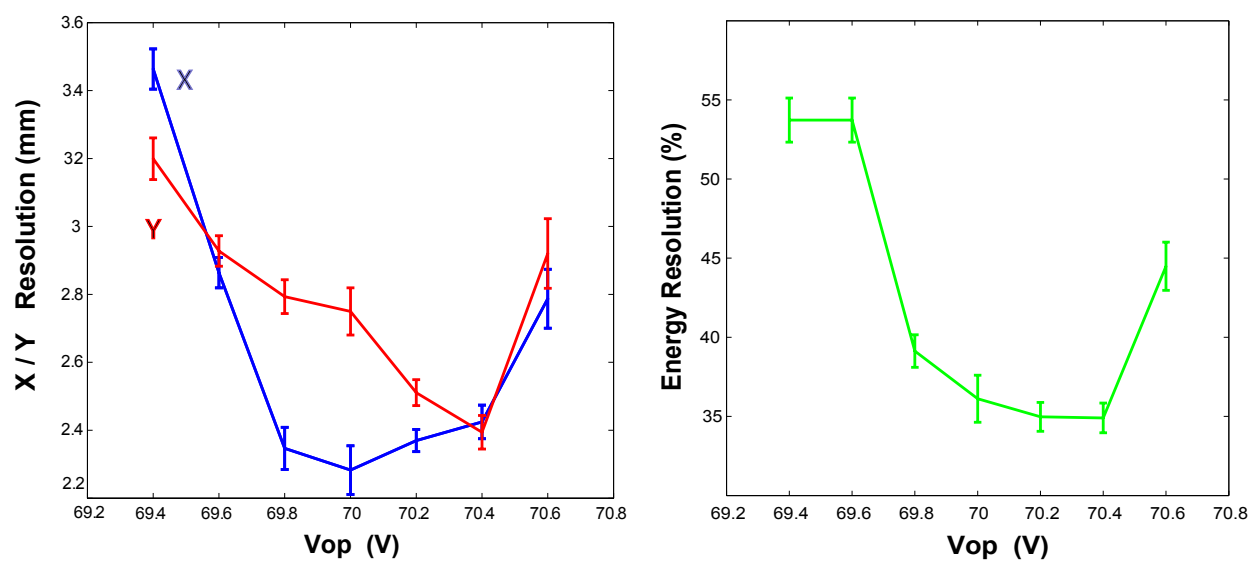

Figure 6. Effect of Operating Voltage (VOP) in detector performance. $(8 \times 8$ array of MPPC S10362-11).

\subsection{SiPM array operating voltage optimization}

AMIC2GR is an integrated front-end with a preamplifier stage optimized for sensor characteristics. As a consequence SNR can be high enough with moderate sensor gain values. This is a useful feature specially with SiPM, since dark noise rises with Operating Voltage (VOP) which in turn must be set to high values if high gains are required. As a consequence spatial and energy resolution have a strong dependence on VOP as shown in figure 6. For low values of VOP, resolution is determined by a low gain which yields a low SNR. High values of VOP produce high dark noise ratios which also degrade SNR and resolution. The differences in X and Y resolution are caused by a non-symmetric dead space distribution due to SiPM packaging structure.

\subsection{SiPM array trigger signal generation}

Another common problem when dealing with arrays of SiPM is to obtain a common trigger signal for timing purposes. AMIC2GR includes a dedicated CB with equalized delays among its inputs 


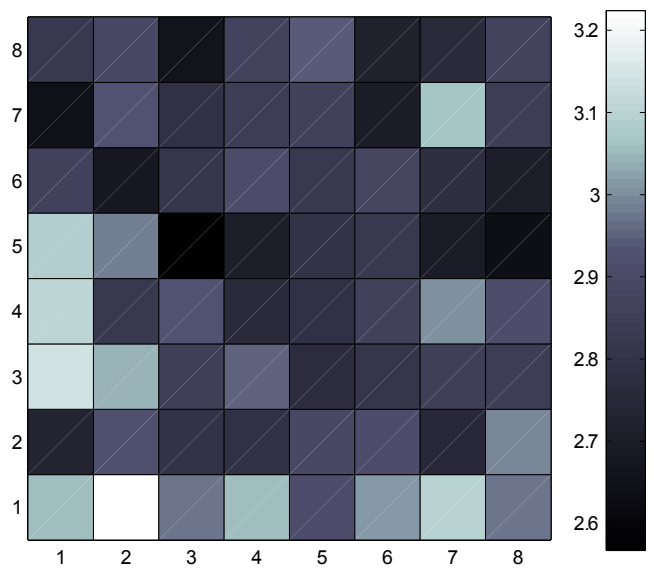

STD. DEVIATION of Time resolution: $114.5 \mathrm{ps}$

Figure 7. Time resolution (ns) vs. XY Position. $(8 \times 8$ array of MPPC S10362-11).

which offers the best characteristics to obtain a good trigger signal as the sum of all the signals of the array. Results of timing resolution spread along detector surface have been measured using the setup structure shown in figure 3a and are presented in figure 7. Absolute values of timing resolution are limited by data acquisition system (DAQ) being used in the test bench. It is a free running sample based DAQ with timestamp generation [6] which offers a best time resolution of $2.5 \mathrm{~ns}$. These measurements include resolution spread due to mismatch among SiPM, PCB routing effects etc.

\section{Conclusions}

A new implementation of AMIC architecture based on AMIC2GR has been presented in this paper. Improved performance of AMIC2GR increases the range of possible photomultipliers which can be used with the same front-end and allows a further enhancement of the detector features. Gain equalization can be easily implemented as well as VOP optimization. Also a common trigger signal can be obtained for SiPM arrays.

\section{Acknowledgments}

This work was supported by Universitat Politècnica de València through research program PAID06-10-2212.

\section{References}

[1] G. Llosa et al., Development of a pet prototype with continuous lyso crystals and monolithic sipm matrices, IEEE NSS/MIC Conf. (2011) 3631.

[2] V. Herrero-Bosch et al., Amic: an expandable front-end for $\gamma$-ray detectors with light distribution analysis capabilities, IEEE Trans. Nucl. Sci. 58 (2011) 1641. 
[3] C. Lerche et al., Depth of $\gamma$-ray interaction within continuous crystals from the width of its scintillation light-distribution, IEEE Trans. Nucl. Sc. 52 (2005) 560.

[4] C. Lerche et al., Fast circuit topology for spatial signal distribution analysis, IEEE NPSS Real Time Conf. Rec. (2010) 1.

[5] K. Bult and G. Geelen, An inherently linear and compact most-only current-division technique, IEEE J. Solid-State Circ. 27 (1992) 1730.

[6] R. Esteve et al., A high performance data acquisitionsystem for a 16-head PET Scanner, talk given at the $11^{\text {th }}$ International Workshop on Radiation Imaging Detectors, June 28-July 2, Prague, Czech Republic (2009). 